\title{
The Global Attractors for a Nonlinear Viscoelastic Wave Equation with Strong Damping and Linear Damping and Source Terms
}

\author{
Liang Guo, Zhaoqin Yuan, Guoguang Lin \\ Department of Mathematics, Yunnan University, Kunming, China \\ Email: guoliang142857@163.com, yuanzq091@163.com, gglin@ynu.edu.cn
}

Received 20 April 2015; accepted 19 June 2015; published 24 June 2015

Copyright (C) 2015 by authors and Scientific Research Publishing Inc.

This work is licensed under the Creative Commons Attribution International License (CC BY). http://creativecommons.org/licenses/by/4.0/

\section{c) (7) Open Access}

\begin{abstract}
In this paper, firstly, some priori estimates are obtained for the existence and uniqueness of solutions of a nonlinear viscoelastic wave equation with strong damping, linear damping and source terms. Then we study the global attractors of the equation.
\end{abstract}

\section{Keywords}

\section{Global Attractors, Viscoelastic Equation, Priori Estimates}

\section{Introduction}

We know that viscoelastic materials have memory effects. These properties are due to the mechanical response influenced by the history of the materials. As these materials have a wide application in the natural science, their dynamics are of great importance and interest. The memory effects can be modeled by a partial differential equation. In recent years, the behaviors of solutions for the PDE system have been studied extensively, and many achievements have been obtained. Many authors have focused on the problem of existence, decay and blow-up for the last two decades, see [1]-[5]. And the attractors are still important contents that are studied.

In [6], R.O. Araújo, T. Ma and Y.M. Qin studied the following equation

$$
\left|u_{t}\right|^{\rho} u_{t t}-\Delta u-\Delta u_{t t}+\int_{0}^{+\infty} g(s) \Delta u(t-s) \mathrm{d} s+f(u)=h(x)
$$

and they proved the global existence, uniqueness and exponential stability of solutions and existence of the 
global attractor.

In [7], Y.M. Qin, B.W. Feng and M. Zhang considered the following initial-boundary value problem:

$$
\left\{\begin{array}{l}
\left|u_{t}\right|^{\rho} u_{t t}-\Delta u-\Delta u_{t t}+\int_{0}^{+\infty} g(s) \Delta u(t-s) \mathrm{d} s+u_{t}=\sigma(x, t), x \in \Omega, t>\tau \\
u(x, t)=0, x \in \partial \Omega, t \geq \tau \\
u(x, \tau)=u_{0}^{\tau}(x), u_{t}(x, \tau)=u_{1}^{\tau}(x), u(x, t)=u_{\tau}(x, t), x \in \Omega, \tau \in R^{+}
\end{array}\right.
$$

where $\Omega$ is a bounded domain of $R^{n}(n \geq 1)$ with a smooth boundary $\partial \Omega, u_{\tau}(x, t)$ (the past history of $u$ ) is a given datum which has to be known for all $t \leq \tau$, the function $g$ represents the kernel of a memory, $\sigma=\sigma(x, t)$ is a non-autonomous term, called a symbol, and $\rho$ is a real number such that $1<\rho \leq \frac{2}{n-2}$ if $n \geq 3 ; \rho>1$ if $n=1,2$. They proved the existence of uniform attractors for a non-autonomous viscoelastic equation with a past history. For more related results, we refer the reader to [8]-[14].

In this work, we intend to study the following initial-boundary problem:

$$
\left\{\begin{array}{l}
u_{t t}-\Delta u+\int_{0}^{+\infty} g(s) \Delta u(t-s) \mathrm{d} s-\varepsilon_{1} \Delta u_{t}+\varepsilon_{2} u_{t}+\varepsilon_{3}|u|^{p-2} u=f(x), \quad x \in \Omega, t>0 \\
u(x, t)=0, \quad x \in \partial \Omega, t \geq 0 \\
u(x, 0)=u_{0}(x), \quad u_{t}(x, 0)=u_{1}(x), \quad x \in \Omega
\end{array}\right.
$$

where $\varepsilon_{1}, \varepsilon_{2}, \varepsilon_{3} \geq 0$, and $\Omega \subset R^{n}(n \geq 1)$ is a bounded domain with smooth boundary $\partial \Omega$, $2<p<\min \left\{\frac{2 n}{n-2}, \frac{2 n+4}{n}\right\}$ if $n \geq 3 ; p>2$ if $n=1,2$, for the problem (1.3), the memory term $\int_{0}^{+\infty} g(s) \Delta u(t-s) \mathrm{d} s$ replaces $\int_{0}^{t} g(t-s) \Delta u(s) \mathrm{d} s$, and we consider the strong damping term $-\varepsilon_{1} \Delta u_{t}$, the linear damping term $\varepsilon_{2} u_{t}$ and source terms $\varepsilon_{3}|u|^{p-2} u$. We define

$$
\eta=\eta(s)=\eta^{t}(x, s)=u(x, t)-u(x, t-s)
$$

A direct computation yields

$$
\eta_{t}(s)=-\eta_{s}(s)+u_{t}(t)
$$

Thus, the original memory term can be written as

$$
\int_{0}^{+\infty} g(s) \Delta u(t-s) \mathrm{d} s=\int_{0}^{+\infty} g(s) \mathrm{d} s \cdot \Delta u-\int_{0}^{+\infty} g(s) \Delta \eta(s) \mathrm{d} s
$$

and we get a new system

$$
\begin{gathered}
u_{t t}-\left(1-\int_{0}^{+\infty} g(s) \mathrm{d} s\right) \Delta u-\varepsilon_{1} \Delta u_{t}-\int_{0}^{+\infty} g(s) \Delta \eta(s) \mathrm{d} s+\varepsilon_{2} u_{t}+\varepsilon_{3}|u|^{p-2} u=f(x) \\
\eta_{t}=-\eta_{s}+u_{t}
\end{gathered}
$$

with the initial conditions

$$
u(x, 0)=u_{0}(x), u_{t}(x, 0)=u_{1}(x), \eta(0)=\eta^{t}(x, 0)=0, x \in \Omega
$$

and the boundary conditions

$$
u(x, t)=0, \quad x \in \partial \Omega, t \geq 0
$$

The rest of this paper is organized as follows. In Section 2, we first obtain the priori estimates, then in Section 3 , we prove the existence of the global attractors. 
For convenience, we denote the norm and scalar product in $L^{2}(\Omega)$ by $\|\cdot\|$ and $(\cdot, \cdot)$, let $V=H^{1}(\Omega)$, $D(A)=H^{2}(\Omega)$.

\section{The Priori Estimates of Solution of Equation}

In this section, we present some materials needed in the proof of our results, state a global existence result, and prove our main result. For this reason, we assume that

(G1) $g: R^{+} \rightarrow R^{+}$is a differentiable function satisfying $1-\int_{0}^{+\infty} g(s) \mathrm{d} s=l>0$;

(G2) $g(s) \geq 0, g^{\prime}(s) \leq 0, \forall s \in R^{+}$;

(G3) There exists a constant $\xi>0$ such that $g^{\prime}(s)+\xi g(s) \leq 0, \quad \forall s \in R^{+}$;

Lemma 1. Assume (G1), (G2) and (G3) hold, let

$$
\begin{cases}2<p<\frac{2 n}{n-2}, & n \geq 3 \\ p \geq 2, & n=1,2\end{cases}
$$

and $\left(u_{0}, u_{1}\right) \in H_{0}^{1}(\Omega) \times L^{2}(\Omega), \quad f \in L^{2}(\Omega), \quad v=u_{t}+\varepsilon u$, then the solution $(u, v)$ of Equation (1.3) satisfies $(u, v) \in H_{0}^{1}(\Omega) \times L^{2}(\Omega)$ and

$$
\|(u, v)\|_{H_{0}^{1} \times L^{2}}^{2}=\|\nabla u\|^{2}+\|v\|_{2}^{2} \leq \frac{W(0)}{k} \mathrm{e}^{-\alpha_{1} t}+\frac{C_{1}}{\alpha_{1} k}\left(1-\mathrm{e}^{-\alpha_{1} t}\right)
$$

here $W(0)=\left\|v_{0}\right\|_{2}^{2}+\left(l-\varepsilon_{1} \varepsilon\right)\left\|\nabla u_{0}\right\|_{2}^{2}+\frac{2 \varepsilon_{3}}{p}\left\|u_{0}\right\|_{p}^{p}$, thus there exists $E_{0}$ and $t_{1}=t_{1}(\Omega)>0$, such that

$$
\|(u, v)\|_{H_{0}^{1} \times L^{2}}^{2}=\|\nabla u(t)\|_{2}^{2}+\|v(t)\|_{2}^{2} \leq E_{0}\left(t>t_{1}\right)
$$

Proof. We multiply $v=u_{t}+\varepsilon u$ with both sides of equation and obtain

$$
\left(u_{t t}-\left(1-\int_{0}^{+\infty} g(s) \mathrm{d} s\right) \Delta u-\varepsilon_{1} \Delta u_{t}-\int_{0}^{+\infty} g(s) \Delta \eta(s) \mathrm{d} s+\varepsilon_{2} u_{t}+\varepsilon_{3}|u|^{p-2} u, v\right)=(f, v)
$$

By using Holder inequality, Young’s inequality and Poincare inequality, we get

$$
\begin{aligned}
\left(u_{t t}, v\right) & =\left(v_{t}-\varepsilon u_{t}, v\right)=\frac{1}{2} \frac{\mathrm{d}}{\mathrm{d} t}\|v\|_{2}^{2}-\varepsilon\left(u_{t}, v\right)=\frac{1}{2} \frac{\mathrm{d}}{\mathrm{d} t}\|v\|_{2}^{2}-\varepsilon(v-\varepsilon u, v) \\
& =\frac{1}{2} \frac{\mathrm{d}}{\mathrm{d} t}\|v\|_{2}^{2}-\varepsilon\|v\|_{2}^{2}+\varepsilon^{2}(u, v) \geq \frac{1}{2} \frac{\mathrm{d}}{\mathrm{d} t}\|v\|_{2}^{2}-\varepsilon\|v\|_{2}^{2}-\frac{\varepsilon^{2}}{2}\|u\|_{2}^{2}-\frac{\varepsilon^{2}}{2}\|v\|_{2}^{2} \\
& \geq \frac{1}{2} \frac{\mathrm{d}}{\mathrm{d} t}\|v\|_{2}^{2}-\varepsilon\|v\|_{2}^{2}-\frac{\varepsilon^{2}}{2 \lambda_{1}}\|\nabla u\|_{2}^{2}-\frac{\varepsilon^{2}}{2}\|v\|_{2}^{2},
\end{aligned}
$$

and

$$
\left(-\left(1-\int_{0}^{+\infty} g(s) \mathrm{d} s\right) \Delta u, v\right)=-l\left(\Delta u, u_{t}+\varepsilon u\right)=\frac{l}{2} \frac{\mathrm{d}}{\mathrm{d} t}\|\nabla u\|_{2}^{2}+l \varepsilon\|\nabla u\|_{2}^{2}
$$

and

$$
\left(-\int_{0}^{+\infty} g(s) \Delta \eta(s) \mathrm{d} s, v\right)=\left(-\int_{0}^{+\infty} g(s) \Delta \eta(s) \mathrm{d} s, u_{t}\right)+\left(-\int_{0}^{+\infty} g(s) \Delta \eta(s) \mathrm{d} s, \varepsilon u\right)
$$

For the first term on the right side (2.5), by using (G1), (G2) and (G3), we have

$$
\begin{aligned}
\int_{0}^{+\infty} g(s) \int_{\Omega} \nabla \eta(s) \cdot \nabla u_{t} \mathrm{~d} x \mathrm{~d} s & =\int_{0}^{+\infty} g(s) \int_{\Omega} \nabla \eta(s) \cdot\left(\nabla \eta_{t}+\nabla \eta_{s}\right) \mathrm{d} x \mathrm{~d} s \\
& =\int_{0}^{+\infty} g(s) \frac{1}{2} \frac{\mathrm{d}}{\mathrm{d} t}\|\nabla \eta\|_{2}^{2} \mathrm{~d} s+\int_{0}^{+\infty} g(s) \mathrm{d} \frac{1}{2}\|\nabla \eta\|_{2}^{2} \\
& \geq \frac{1}{2} \frac{\mathrm{d}}{\mathrm{d} t}\|\eta\|_{g, V}^{2}+\frac{\xi}{2}\|\eta\|_{g, V}^{2},
\end{aligned}
$$


where

$$
\|\eta\|_{g, V}^{2}=\int_{0}^{+\infty} g(s)\|\nabla \eta(s)\|_{2}^{2} \mathrm{~d} s
$$

For the second term on the right side (2.5), by using Holder inequality and Young's inequality, we get

$$
\begin{aligned}
\left(-\int_{0}^{+\infty} g(s) \Delta \eta(s) \mathrm{d} s, \varepsilon u\right) & =\varepsilon \int_{0}^{+\infty} g(s) \int_{\Omega} \nabla \eta(s) \nabla u \mathrm{~d} x \mathrm{~d} s \\
& \geq-\frac{\xi}{4}\|\eta\|_{g, V}^{2}-\frac{\varepsilon^{2}}{\xi} \int_{0}^{+\infty} g(s) \mathrm{d} s\|\nabla u\|_{2}^{2}
\end{aligned}
$$

So, we have

$$
\begin{aligned}
\left(-\int_{0}^{+\infty} g(s) \Delta \eta(s) \mathrm{d} s, v\right) & =\left(-\int_{0}^{+\infty} g(s) \Delta \eta(s) \mathrm{d} s, u_{t}\right)+\left(-\int_{0}^{+\infty} g(s) \Delta \eta(s) \mathrm{d} s, \varepsilon u\right) \\
& \geq \frac{1}{2} \frac{\mathrm{d}}{\mathrm{d} t}\|\eta\|_{g, V}^{2}+\frac{\xi}{4}\|\eta\|_{g, V}^{2}-\frac{\varepsilon^{2}}{\xi} \int_{0}^{+\infty} g(s) \mathrm{d} s\|\nabla u\|_{2}^{2}
\end{aligned}
$$

By using Poincare inequality, we obtain

$$
\begin{aligned}
\left(-\varepsilon_{1} \Delta u_{t}, v\right) & =\varepsilon_{1}(-\Delta v+\varepsilon \Delta u, v)=\varepsilon_{1}\|\nabla v\|_{2}^{2}+\varepsilon_{1} \varepsilon\left(\Delta u, u_{t}+\varepsilon u\right) \\
& =\varepsilon_{1}\|\nabla v\|_{2}^{2}-\frac{\varepsilon_{1} \varepsilon}{2} \frac{\mathrm{d}}{\mathrm{d} t}\|\nabla u\|_{2}^{2}-\varepsilon_{1} \varepsilon^{2}\|\nabla u\|_{2}^{2} \\
& \geq \varepsilon_{1} \lambda_{1}\|v\|_{2}^{2}-\frac{\varepsilon_{1} \varepsilon}{2} \frac{\mathrm{d}}{\mathrm{d} t}\|\nabla u\|_{2}^{2}-\varepsilon_{1} \varepsilon^{2}\|\nabla u\|_{2}^{2}
\end{aligned}
$$

and

$$
\begin{aligned}
\left(\varepsilon_{2} u_{t}, v\right) & =\varepsilon_{2}\left(u_{t}, u_{t}+\varepsilon u\right)=\varepsilon_{2}\left\|u_{t}\right\|_{2}^{2}-\varepsilon_{2} \varepsilon\|u\|_{2}\left\|u_{t}\right\|_{2} \\
& \geq \varepsilon_{2}\left\|u_{t}\right\|_{2}^{2}-\frac{\varepsilon_{2} \varepsilon}{2}\|u\|_{2}^{2}-\frac{\varepsilon_{2} \varepsilon}{2}\left\|u_{t}\right\|_{2}^{2} \\
& \geq \varepsilon_{2}\left(1-\frac{\varepsilon}{2}\right)\left\|u_{t}\right\|_{2}^{2}-\frac{\varepsilon_{2} \varepsilon}{2 \lambda_{1}}\|\nabla u\|_{2}^{2}
\end{aligned}
$$

and

$$
\left(\varepsilon_{3}|u|^{p-2} u, v\right)=\varepsilon_{3}\left(|u|^{p-2} u, u_{t}+\varepsilon u\right)=\frac{\varepsilon_{3}}{p} \frac{\mathrm{d}}{\mathrm{d} t}\|u\|_{p}^{p}+\varepsilon_{3} \varepsilon\|u\|_{p}^{p}
$$

By using Holder inequality and Young's inequality, we obtain

$$
(f(x), v) \leq\|f\| \cdot\|v\| \leq \frac{\lambda_{1}}{2}\|v\|_{2}^{2}+\frac{1}{2 \lambda_{1}}\|f\|_{2}^{2}
$$

Then, we have

$$
\begin{aligned}
& \frac{1}{2} \frac{\mathrm{d}}{\mathrm{d} t}\|v\|_{2}^{2}-\varepsilon\|v\|_{2}^{2}-\frac{\varepsilon^{2}}{2 \lambda_{1}}\|\nabla u\|_{2}^{2}-\frac{\varepsilon^{2}}{2}\|v\|_{2}^{2}+\frac{l}{2} \frac{\mathrm{d}}{\mathrm{d} t}\|\nabla u\|_{2}^{2}+l \varepsilon\|\nabla u\|_{2}^{2}+\frac{1}{2} \frac{\mathrm{d}}{\mathrm{d} t}\|\eta\|_{g, V}^{2} \\
& +\frac{\xi}{4}\|\eta\|_{g, V}^{2}-\frac{\varepsilon^{2}}{\xi} \int_{0}^{+\infty} g(s) \mathrm{d} s\|\nabla u\|_{2}^{2}+\varepsilon_{1} \lambda_{1}\|v\|_{2}^{2}-\frac{\varepsilon_{1} \varepsilon}{2} \frac{\mathrm{d}}{\mathrm{d} t}\|\nabla u\|_{2}^{2}-\varepsilon_{1} \varepsilon^{2}\|\nabla u\|_{2}^{2} \\
& +\varepsilon_{2}\left(1-\frac{\varepsilon}{2}\right)\left\|u_{t}\right\|_{2}^{2}-\frac{\varepsilon_{2} \varepsilon}{2 \lambda_{1}}\|\nabla u\|_{2}^{2}+\frac{\varepsilon_{3}}{p} \frac{\mathrm{d}}{\mathrm{d} t}\|u\|_{p}^{p}+\varepsilon_{3} \varepsilon\|u\|_{p}^{p} \\
& \leq \frac{\lambda_{1}}{2}\|v\|_{2}^{2}+\frac{1}{2 \lambda_{1}}\|f\|_{2}^{2}
\end{aligned}
$$


That is

$$
\begin{aligned}
& \frac{\mathrm{d}}{\mathrm{d} t}\left[\|v\|_{2}^{2}+\left(l-\varepsilon_{1} \varepsilon\right)\|\nabla u\|_{2}^{2}+\|\eta\|_{g, V}^{2}+\frac{2 \varepsilon_{3}}{p}\|u\|_{p}^{p}\right]+\left(2 \varepsilon_{1} \lambda_{1}-2 \varepsilon-\varepsilon^{2}-\lambda_{1}\right)\|v\|_{2}^{2} \\
& +2 \varepsilon\left(l-\varepsilon_{1} \varepsilon-\frac{\varepsilon}{2 \lambda_{1}}-\frac{\varepsilon}{\xi} \int_{0}^{+\infty} g(s) \mathrm{d} s-\frac{\varepsilon_{2}}{2 \lambda_{1}}\right)\|\nabla u\|_{2}^{2}+\frac{\xi}{2}\|\eta\|_{g, V}^{2}+2 \varepsilon_{3} \varepsilon\|u\|_{p}^{p} \\
& +2 \varepsilon_{2}\left[1-\frac{\varepsilon}{2}\right]\left\|u_{t}\right\|_{2}^{2} \leq \frac{1}{\lambda_{1}}\|f\|_{2}^{2}
\end{aligned}
$$

Next, we take proper $\varepsilon, \varepsilon_{1}, \varepsilon_{2}, \varepsilon_{3}$, such that

$$
\left\{\begin{array}{l}
a_{1}=2 \varepsilon_{1} \lambda_{1}-2 \varepsilon-\varepsilon^{2}-\lambda_{1} \geq 0 \\
a_{2}=2 \varepsilon\left(l-\varepsilon_{1} \varepsilon-\frac{\varepsilon}{2 \lambda_{1}}-\frac{\varepsilon}{\xi} \int_{0}^{+\infty} g(s) \mathrm{d} s-\frac{\varepsilon_{2}}{2 \lambda_{1}}\right) \geq 0 \\
a_{3}=2 \varepsilon_{2}\left(1-\frac{\varepsilon}{2}\right) \geq 0
\end{array}\right.
$$

Taking $\alpha_{1}=\min \left\{a_{1}, \frac{a_{2}}{l-\varepsilon_{1} \varepsilon}, \frac{\xi}{2}, p \varepsilon\right\}$, then

$$
\frac{\mathrm{d}}{\mathrm{d} t} W(t)+\alpha_{1} W(t) \leq \frac{1}{\lambda_{1}}\|f\|_{2}^{2}:=C_{1}
$$

where $W(t)=\|v\|_{2}^{2}+\left(l-\varepsilon_{1} \varepsilon\right)\|\nabla u\|_{2}^{2}+\|\eta\|_{g, V}^{2}+\frac{2 \varepsilon_{3}}{p}\|u\|_{p}^{p}$, by using Gronwall inequality, we obtain

$$
W(t) \leq W(0) \mathrm{e}^{-\alpha_{1} t}+\frac{C_{1}}{\alpha_{1}}\left(1-\mathrm{e}^{-\alpha_{1} t}\right)
$$

From $2<p<\frac{2 n}{n-2}, n \geq 3$, according to Embedding Theorem then $H_{0}^{1}(\Omega) \subset L^{p}(\Omega)$, let $k=\min \left\{1,\left(l-\varepsilon_{1} \varepsilon\right)\right\}$, so we have

$$
\|(u, v)\|_{H_{0}^{1} \times L^{2}}^{2}=\|\nabla u\|^{2}+\|v\|_{2}^{2} \leq \frac{W(0)}{k} \mathrm{e}^{-\alpha_{1} t}+\frac{C_{1}}{\alpha_{1} k}\left(1-\mathrm{e}^{-\alpha_{1} t}\right)
$$

Then

$$
\varlimsup_{t \rightarrow \infty}\|(u, v)\|_{H_{0}^{1} \times L^{2}}^{2} \leq \frac{C_{1}}{\alpha_{1} k}
$$

So, there exists $E_{0}$ and $t_{1}=t_{1}(\Omega)>0$, such that

$$
\|(u, v)\|_{H_{0}^{1} \times L^{2}}^{2}=\|\nabla u(t)\|_{2}^{2}+\|v\|_{2}^{2} \leq E_{0}\left(t>t_{1}\right)
$$

Lemma 2. Assume (G1), (G2) and (G3) hold, let

$$
\begin{cases}2<p<\frac{2 n+4}{n}, & n \geq 3 \\ p \geq 2, & n=1,2\end{cases}
$$


and $\left(u_{0}, u_{1}\right) \in H^{2}(\Omega) \times H^{1}(\Omega), \quad f \in H^{1}(\Omega), \quad v=u_{t}+\varepsilon u$, then the solution $(u, v)$ of Equation (1.3) satisfies $(u, v) \in H^{2}(\Omega) \times H^{1}(\Omega)$ and

$$
\|(u, v)\|_{H_{0}^{2} \times H^{1}}^{2}=\|\Delta u\|_{2}^{2}+\|\nabla v\|_{2}^{2} \leq \frac{W(0)}{k} \mathrm{e}^{-\alpha_{2} t}+\frac{C_{2}}{\alpha_{2} k}\left(1-\mathrm{e}^{-\alpha_{2} t}\right)
$$

Here $V(0)=\left\|\nabla u_{1}+\nabla u_{0}\right\|_{2}^{2}+\left(l-\varepsilon_{1} \varepsilon\right)\left\|\Delta u_{0}\right\|_{2}^{2}$, thus there exists $E_{1}$ and $t_{2}=t_{2}(\Omega)>0$, such that

$$
\|(u, v)\|_{H^{2} \times H^{1}}^{2}=\|\Delta u(t)\|_{2}^{2}+\|\nabla v(t)\|_{2}^{2} \leq E_{1}\left(t>t_{2}\right)
$$

Proof. We multiply $-\Delta v=-\Delta u_{t}-\varepsilon \Delta u$ with both sides of equation and obtain

$$
\left(u_{t t}-\left(1-\int_{0}^{+\infty} g(s) \mathrm{d} s\right) \Delta u-\varepsilon_{1} \Delta u_{t}-\int_{0}^{+\infty} g(s) \Delta \eta(s) \mathrm{d} s+\varepsilon_{2} u_{t}+\varepsilon_{3}|u|^{p-2} u,-\Delta v\right)=(f,-\Delta v)
$$

By using Holder inequality, Young’s inequality and Poincare inequality, we get

$$
\begin{aligned}
\left(u_{t t},-\Delta v\right) & =\left(v_{t}-\varepsilon u_{t},-\Delta v\right)=\frac{1}{2} \frac{\mathrm{d}}{\mathrm{d} t}\|\nabla v\|_{2}^{2}-\varepsilon\left(u_{t},-\Delta v\right) \\
& =\frac{1}{2} \frac{\mathrm{d}}{\mathrm{d} t}\|\nabla v\|_{2}^{2}-\varepsilon(v-\varepsilon u,-\Delta v) \\
& =\frac{1}{2} \frac{\mathrm{d}}{\mathrm{d} t}\|\nabla v\|_{2}^{2}-\varepsilon\|\nabla v\|_{2}^{2}+\varepsilon^{2}(\nabla u, \nabla v) \\
& \geq \frac{1}{2} \frac{\mathrm{d}}{\mathrm{d} t}\|\nabla v\|_{2}^{2}-\varepsilon\|\nabla v\|_{2}^{2}-\frac{\varepsilon^{2}}{2}\|\nabla u\|_{2}^{2}-\frac{\varepsilon^{2}}{2}\|\nabla v\|_{2}^{2} \\
& \geq \frac{1}{2} \frac{\mathrm{d}}{\mathrm{d} t}\|\nabla v\|_{2}^{2}-\varepsilon\|\nabla v\|_{2}^{2}-\frac{\varepsilon^{2}}{2 \lambda_{1}}\|\Delta u\|_{2}^{2}-\frac{\varepsilon^{2}}{2}\|\nabla v\|_{2}^{2}
\end{aligned}
$$

and

$$
\left(-\left(1-\int_{0}^{+\infty} g(s) \mathrm{d} s\right) \Delta u,-\Delta v\right)=-l\left(\Delta u,-\Delta u_{t}-\varepsilon \Delta u\right)=\frac{l}{2} \frac{\mathrm{d}}{\mathrm{d} t}\|\Delta u\|_{2}^{2}+l \varepsilon\|\Delta u\|_{2}^{2}
$$

and

$$
\left(-\int_{0}^{+\infty} g(s) \Delta \eta(s) \mathrm{d} s,-\Delta v\right)=\left(-\int_{0}^{+\infty} g(s) \Delta \eta(s) \mathrm{d} s,-\Delta u_{t}\right)+\left(-\int_{0}^{+\infty} g(s) \Delta \eta(s) \mathrm{d} s,-\varepsilon \Delta u\right)
$$

For the first term on the right side (2.23), by using (G1), (G2) and (G3), we have

$$
\begin{aligned}
\int_{0}^{+\infty} g(s) \int_{\Omega} \Delta \eta(s) \cdot \Delta u_{t} \mathrm{~d} x \mathrm{~d} s & =\int_{0}^{+\infty} g(s) \int_{\Omega} \Delta \eta(s) \cdot\left(\Delta \eta_{t}+\Delta \eta_{s}\right) \mathrm{d} x \mathrm{~d} s \\
& =\int_{0}^{+\infty} g(s) \frac{1}{2} \frac{\mathrm{d}}{\mathrm{d} t}\|\Delta \eta\|_{2}^{2} \mathrm{~d} s+\int_{0}^{+\infty} g(s) \mathrm{d} \frac{1}{2}\|\Delta \eta\|_{2}^{2} \\
& \geq \frac{1}{2} \frac{\mathrm{d}}{\mathrm{d} t}\|\eta\|_{g, D(A)}^{2}+\frac{\xi}{2}\|\eta\|_{g, D(A)}^{2}
\end{aligned}
$$

where

$$
\|\eta\|_{g, D(A)}^{2}=\int_{0}^{+\infty} g(s)\|\Delta \eta(s)\|_{2}^{2} \mathrm{~d} s
$$

For the second term on the right side (2.23), by using Holder inequality and Young's inequality, we get

$$
\begin{aligned}
\left(-\int_{0}^{+\infty} g(s) \Delta \eta(s) \mathrm{d} s,-\varepsilon \Delta u\right) & =\varepsilon \int_{0}^{+\infty} g(s) \int_{\Omega} \Delta \eta(s) \Delta u \mathrm{~d} x \mathrm{~d} s \\
& \geq-\frac{\xi}{4}\|\eta\|_{g, D(A)}^{2}-\frac{\varepsilon^{2}}{\xi} \int_{0}^{+\infty} g(s) \mathrm{d} s\|\Delta u\|_{2}^{2}
\end{aligned}
$$

so, we have 


$$
\begin{aligned}
& \left(-\int_{0}^{+\infty} g(s) \Delta \eta(s) \mathrm{d} s,-\Delta v\right) \\
& =\left(-\int_{0}^{+\infty} g(s) \Delta \eta(s) \mathrm{d} s,-\Delta u_{t}\right)+\left(-\int_{0}^{+\infty} g(s) \Delta \eta(s) \mathrm{d} s,-\varepsilon \Delta u\right) \\
& =\frac{1}{2} \frac{\mathrm{d}}{\mathrm{d} t}\|\eta\|_{g, D(A)}^{2}+\frac{\xi}{4}\|\eta\|_{g, D(A)}^{2}-\frac{\varepsilon^{2}}{\xi} \int_{0}^{+\infty} g(s) \mathrm{d} s\|\Delta u\|_{2}^{2}
\end{aligned}
$$

By using Poincare inequality, we have

$$
\begin{aligned}
\left(-\varepsilon_{1} \Delta u_{t},-\Delta v\right) & =\varepsilon_{1}(-\Delta v+\varepsilon \Delta u,-\Delta v)=\varepsilon_{1}\|\Delta v\|_{2}^{2}+\varepsilon_{1} \varepsilon\left(\Delta u,-\Delta u_{t}-\varepsilon \Delta u\right) \\
& =\varepsilon_{1}\|\Delta v\|_{2}^{2}-\frac{\varepsilon_{1} \varepsilon}{2} \frac{\mathrm{d}}{\mathrm{d} t}\|\Delta u\|_{2}^{2}-\varepsilon_{1} \varepsilon^{2}\|\Delta u\|_{2}^{2},
\end{aligned}
$$

and

$$
\begin{aligned}
\left(\varepsilon_{2} u_{t},-\Delta v\right) & =\varepsilon_{2}\left(u_{t},-\Delta u_{t}-\varepsilon \Delta u\right)=\varepsilon_{2}\left\|\nabla u_{t}\right\|_{2}^{2}-\varepsilon_{2} \varepsilon\|\nabla u\|_{2}\left\|\nabla u_{t}\right\|_{2} \\
& \geq \varepsilon_{2}\left(1-\frac{\varepsilon}{2}\right)\left\|\nabla u_{t}\right\|_{2}^{2}-\frac{\varepsilon_{2} \varepsilon}{2 \lambda_{1}}\|\Delta u\|_{2}^{2}
\end{aligned}
$$

And using Interpolation Theorem, we have

$$
\begin{aligned}
\left(\varepsilon_{3}|u|^{p-2} u,-\Delta v\right) & \leq \varepsilon_{3}\|u\|_{2 p-2}^{p-1}\|\Delta v\|_{2} \leq \varepsilon_{3} C_{0}\left(\|u\|_{2}\right)\|\Delta u\|_{2}^{\frac{n(p-2)}{4}}\|\Delta v\|_{2} \\
& \leq \frac{\varepsilon_{1}}{2}\|\Delta v\|_{2}^{2}+\frac{\varepsilon_{3}^{2} C_{0}^{2}\left(\|u\|_{2}\right)}{2 \varepsilon_{1}}\|\Delta u\|_{2}^{\frac{n(p-2)}{2}} \\
& \leq \frac{\varepsilon_{1}}{2}\|\Delta v\|_{2}^{2}+\frac{l \varepsilon}{2}\|\Delta u\|_{2}^{2}+C_{0}\left(\|u\|_{2}, \varepsilon_{1}, \varepsilon_{3}, l, \varepsilon\right) .
\end{aligned}
$$

By using Holder inequality and Young's inequality, we have

$$
(f(x),-\Delta v) \leq\|\nabla f\| \cdot\|\nabla v\| \leq \frac{\lambda_{1} \varepsilon_{1}}{4}\|\nabla v\|_{2}^{2}+\frac{1}{\lambda_{1} \varepsilon_{1}}\|\nabla f\|_{2}^{2},
$$

Then, we have

$$
\begin{aligned}
& \frac{1}{2} \frac{\mathrm{d}}{\mathrm{d} t}\|\nabla v\|_{2}^{2}-\varepsilon\|\nabla v\|_{2}^{2}-\frac{\varepsilon^{2}}{2 \lambda_{1}}\|\Delta u\|_{2}^{2}-\frac{\varepsilon^{2}}{2}\|\nabla v\|_{2}^{2}+\frac{l}{2} \frac{\mathrm{d}}{\mathrm{d} t}\|\Delta u\|_{2}^{2}+l \varepsilon\|\Delta u\|_{2}^{2} \\
& +\frac{1}{2} \frac{\mathrm{d}}{\mathrm{d} t}\|\eta\|_{g, D(A)}^{2}+\frac{\xi}{4}\|\eta\|_{g, D(A)}^{2}-\frac{\varepsilon^{2}}{\xi} \int_{0}^{+\infty} g(s) \mathrm{d} s\|\Delta u\|_{2}^{2}+\varepsilon_{1}\|\Delta v\|_{2}^{2} \\
& -\frac{\varepsilon_{1} \varepsilon}{2} \frac{\mathrm{d}}{\mathrm{d} t}\|\Delta u\|_{2}^{2}-\varepsilon_{1} \varepsilon^{2}\|\Delta u\|_{2}^{2}+\varepsilon_{2}\left(1-\frac{\varepsilon}{2}\right)\left\|\nabla u_{t}\right\|_{2}^{2}-\frac{\varepsilon_{2} \varepsilon}{2 \lambda_{1}}\|\Delta u\|_{2}^{2} \\
& \leq \frac{\varepsilon_{1}}{2}\|\Delta v\|_{2}^{2}+\frac{l \varepsilon}{2}\|\Delta u\|_{2}^{2}+C_{0}\left(\|u\|_{2}, \varepsilon_{1}, \varepsilon_{3}, l, \varepsilon\right)+\frac{\lambda_{1} \varepsilon_{1}}{4}\|\nabla v\|_{2}^{2}+\frac{1}{\lambda_{1} \varepsilon_{1}}\|\nabla f\|_{2}^{2}
\end{aligned}
$$

That is

$$
\begin{aligned}
& \frac{\mathrm{d}}{\mathrm{d} t}\left[\|\nabla v\|_{2}^{2}+\left(l-\varepsilon_{1} \varepsilon\right)\|\Delta u\|_{2}^{2}+\|\eta\|_{g, D(A)}^{2}\right]+\left(\frac{\varepsilon_{1} \lambda_{1}}{2}-2 \varepsilon-\varepsilon^{2}\right)\|\nabla v\|_{2}^{2} \\
& +2 \varepsilon\left(\frac{l}{2}-\varepsilon_{1} \varepsilon-\frac{\varepsilon}{2 \lambda_{1}}-\frac{\varepsilon}{\xi} \int_{0}^{+\infty} g(s) \mathrm{d} s-\frac{\varepsilon_{2}}{2 \lambda_{1}}\right)\|\Delta u\|_{2}^{2}+\frac{\xi}{2}\|\eta\|_{g, D(A)}^{2} \\
& +2 \varepsilon_{2}\left[1-\frac{\varepsilon}{2}\right]\left\|\nabla u_{t}\right\|_{2}^{2} \leq \frac{2}{\lambda_{1} \varepsilon_{1}}\|\nabla f\|_{2}^{2}+2 C_{0}\left(\|u\|_{2}, \varepsilon_{1}, \varepsilon_{3}, l, \varepsilon\right) .
\end{aligned}
$$


Next, we take proper $\varepsilon, \varepsilon_{1}, \varepsilon_{2}, \varepsilon_{3}$, such that

$$
\left\{\begin{array}{l}
b_{1}=\frac{\varepsilon_{1} \lambda_{1}}{2}-2 \varepsilon-\varepsilon^{2} \geq 0 \\
b_{2}=2 \varepsilon\left(\frac{l}{2}-\varepsilon_{1} \varepsilon-\frac{\varepsilon}{2 \lambda_{1}}-\frac{\varepsilon}{\xi} \int_{0}^{+\infty} g(s) \mathrm{d} s-\frac{\varepsilon_{2}}{2 \lambda_{1}}\right) \geq 0 \\
b_{3}=2 \varepsilon_{2}\left(1-\frac{\varepsilon}{2}\right) \geq 0
\end{array}\right.
$$

Taking $\alpha_{2}=\min \left\{b_{1}, \frac{b_{2}}{l-\varepsilon_{1} \varepsilon}, \frac{\xi}{2}\right\}$, then

$$
\frac{\mathrm{d}}{\mathrm{d} t} V(t)+\alpha_{2} V(t) \leq \frac{2}{\lambda_{1} \varepsilon_{1}}\|\nabla f\|_{2}^{2}+2 C_{0}\left(\|u\|_{2}, \varepsilon_{1}, \varepsilon_{3}, l, \varepsilon\right):=C_{2},
$$

where $V(t)=\|\nabla v\|_{2}^{2}+\left(l-\varepsilon_{1} \varepsilon\right)\|\Delta u\|_{2}^{2}+\|\eta\|_{g, D(A)}^{2}$, by Gronwall inequality, we have

$$
V(t) \leq V(0) \mathrm{e}^{-\alpha_{2} t}+\frac{C_{2}}{\alpha_{2}}\left(1-\mathrm{e}^{-\alpha_{2} t}\right)
$$

From $2<p \leq \frac{2 n}{n-2}$, according to Embedding Theorem, then $H^{2}(\Omega) \subset W^{1, p}(\Omega)$, let $k=\min \left\{1,\left(l-\varepsilon_{1} \varepsilon\right)\right\}$, so, we have

$$
\|(u, v)\|_{H^{2} \times H^{1}}^{2}=\|\Delta u\|_{2}^{2}+\|\nabla v\|_{2}^{2} \leq \frac{V(0)}{k} \mathrm{e}^{-\alpha_{2} t}+\frac{C_{2}}{\alpha_{2} k}\left(1-\mathrm{e}^{-\alpha_{2} t}\right)
$$

then

$$
\varlimsup_{t \rightarrow \infty}\|(u, v)\|_{H^{2} \times H^{1}}^{2} \leq \frac{C_{2}}{\alpha_{2} k}
$$

So, there exists $E_{1}>0$ and $t_{2}=t_{2}(\Omega)>0$, such that

$$
\|(u, v)\|_{H^{2} \times H^{1}}^{2}=\|\Delta u(t)\|_{2}^{2}+\|\nabla v(t)\|_{2}^{2} \leq E_{1}\left(t>t_{2}\right) .
$$

\section{Global Attractors}

Theorem 1. Assume (G1), (G2) and (G3) hold, let

$$
\begin{cases}2<p<\min \left\{\frac{2 n}{n-2}, \frac{2 n+4}{n}\right\}, & n \geq 3 \\ p \geq 2, & n=1,2\end{cases}
$$

and $\left(u_{0}, u_{1}\right) \in H^{2}(\Omega) \times H^{1}(\Omega), \quad f \in H^{1}(\Omega), \quad v=u_{t}+\varepsilon u$, so Equation (1.3) exists a unique smooth solution

$$
(u, v) \in L^{\infty}\left([0,+\infty) ; H^{2}(\Omega) \times H^{1}(\Omega)\right)
$$

Proof. By the method of Galerkin and Lemma 1 and Lemma 2, we can easily obtain the existence of solutions. Next, we prove the uniqueness of solutions in detail.

Assume that $u, v$ are two solutions of equation, let $w=u-v$, then, the two equations subtract and obtain

$$
\begin{aligned}
& w^{\prime \prime}-\left(1-\int_{0}^{+\infty} g(s) \mathrm{d} s\right) \Delta w-\int_{0}^{+\infty} g(s)\left(\Delta \eta_{1}-\Delta \eta_{2}\right) \mathrm{d} s-\varepsilon_{1} \Delta w^{\prime}+\varepsilon_{2} w^{\prime} \\
& =\varepsilon_{3}\left(|v|^{p-2} v-|u|^{p-2} u\right)
\end{aligned}
$$


where

$$
\eta_{1}=u(x, t)-u(x, t-s), \eta_{2}=v(x, t)-v(x, t-s)
$$

By multiplying the equation by $w^{\prime}$ and integrating over $\Omega$, we get

$$
\begin{aligned}
& \left(w^{\prime \prime}-\left(1-\int_{0}^{+\infty} g(s) \mathrm{d} s\right) \Delta w-\int_{0}^{+\infty} g(s)\left(\Delta \eta_{1}-\Delta \eta_{2}\right) \mathrm{d} s-\varepsilon_{1} \Delta w^{\prime}+\varepsilon_{2} w^{\prime}, w^{\prime}\right) \\
& =\left(\varepsilon_{3}\left(|v|^{p-2} v-|u|^{p-2} u\right), w^{\prime}\right)
\end{aligned}
$$

here

$$
\left(w^{\prime \prime}, w^{\prime}\right)=\frac{1}{2} \frac{\mathrm{d}}{\mathrm{d} t}\left\|w^{\prime}\right\|_{2}^{2}
$$

and

$$
\left(-\left(1-\int_{0}^{+\infty} g(s) \mathrm{d} s\right) \Delta w, w^{\prime}\right)=\frac{l}{2} \frac{\mathrm{d}}{\mathrm{d} t}\|\nabla w\|_{2}^{2}
$$

by using (G1), (G2) and (G3), we have

$$
\begin{aligned}
\left(-\int_{0}^{+\infty} g(s)\left(\Delta \eta_{1}-\Delta \eta_{2}\right) \mathrm{d} s, w^{\prime}\right) & =\left(-\int_{0}^{+\infty} g(s)\left(\Delta \eta_{1}-\Delta \eta_{2}\right) \mathrm{d} s,\left(\eta_{1}-\eta_{2}\right)_{t}+\left(\eta_{1}-\eta_{2}\right)_{s}\right) \\
& \geq \frac{1}{2} \frac{\mathrm{d}}{\mathrm{d} t}\left\|\eta_{1}-\eta_{2}\right\|_{g, V}^{2}+\frac{\xi}{2}\left\|\eta_{1}-\eta_{2}\right\|_{g, V}^{2}
\end{aligned}
$$

By using Poincare inequality, we have

$$
\left(-\varepsilon_{1} \Delta w^{\prime}, w^{\prime}\right)=\varepsilon_{1}\left\|\nabla w^{\prime}\right\|_{2}^{2} \geq \varepsilon_{1} \lambda_{1}\left\|w^{\prime}\right\|_{2}^{2}
$$

and

$$
\left(\varepsilon_{2} w^{\prime}, w^{\prime}\right)=\varepsilon_{2}\left\|w^{\prime}\right\|_{2}^{2}
$$

By using Holder inequality, Young's inequality and Poincare inequality, we have

$$
\begin{aligned}
& \varepsilon_{3}\left(|u|^{p-2} u-|v|^{p-2} v, w^{\prime}\right)=\varepsilon_{2} \int\left(|u|^{p-2} u-|v|^{p-2} v\right) w^{\prime} \mathrm{d} x \\
& \leq \varepsilon_{2} p \int\left(|u|^{p-1}+|v|^{p-1}\right)\left|w\left\|w^{\prime} \mid \mathrm{d} x \leq \varepsilon_{3} C_{0}\right\| w\|\| w^{\prime} \|\right. \\
& \leq 2 \varepsilon_{3} \lambda_{1}\left\|w^{\prime}\right\|_{2}^{2}+\frac{\varepsilon_{3} C_{0}^{2}}{8 \lambda_{1}}\|w\|_{2}^{2} \leq 2 \varepsilon_{3} \lambda_{1}\left\|w^{\prime}\right\|_{2}^{2}+\frac{\varepsilon_{3} C_{0}^{2}}{8 \lambda_{1}^{2}}\|\nabla w\|_{2}^{2}
\end{aligned}
$$

then, we have

$$
\begin{aligned}
& \frac{1}{2} \frac{\mathrm{d}}{\mathrm{d} t}\left\|w^{\prime}\right\|_{2}^{2}+\frac{l}{2} \frac{\mathrm{d}}{\mathrm{d} t}\|\nabla w\|_{2}^{2}+\frac{1}{2} \frac{\mathrm{d}}{\mathrm{d} t}\left\|\eta_{1}-\eta_{2}\right\|_{g, V}^{2}+\frac{\xi}{2}\left\|\eta_{1}-\eta_{2}\right\|_{g, V}^{2}+\varepsilon_{1} \lambda_{1}\left\|w^{\prime}\right\|_{2}^{2}+\varepsilon_{2}\left\|w^{\prime}\right\|_{2}^{2} \\
& \leq 2 \varepsilon_{3} \lambda_{1}\left\|w^{\prime}\right\|_{2}^{2}+\frac{\varepsilon_{3} C_{0}^{2}}{8 \lambda_{1}^{2}}\|\nabla w\|_{2}^{2}
\end{aligned}
$$

That is

$$
\frac{\mathrm{d}}{\mathrm{d} t}\left[\left\|w^{\prime}\right\|_{2}^{2}+l\|\nabla w\|_{2}^{2}+\left\|\eta_{1}-\eta_{2}\right\|_{g, V}^{2}\right] \leq 4 \varepsilon_{3} \lambda_{1}\left\|w^{\prime}\right\|_{2}^{2}+\frac{\varepsilon_{3} C_{0}^{2}}{4 \lambda_{1}^{2}}\|\nabla w\|_{2}^{2}+\left\|\eta_{1}-\eta_{2}\right\|_{g, V}^{2}
$$

Taking $m=\max \left\{4 \varepsilon_{3} \lambda_{1}, \frac{\varepsilon_{3} C_{0}^{2}}{4 \lambda_{1}^{2} l}, 1\right\}$, then 


$$
\frac{\mathrm{d}}{\mathrm{d} t}\left[\left\|w^{\prime}\right\|_{2}^{2}+l\|\nabla w\|_{2}^{2}+\left\|\eta_{1}-\eta_{2}\right\|_{g, V}^{2}\right] \leq m\left(\left\|w^{\prime}\right\|_{2}^{2}+l\|\nabla w\|_{2}^{2}+\left\|\eta_{1}-\eta_{2}\right\|_{g, V}^{2}\right)
$$

By using Gronwall inequality, we have

$$
\left\|w^{\prime}\right\|_{2}^{2}+l\|\nabla w\|_{2}^{2}+\left\|\eta_{1}-\eta_{2}\right\|_{g, V}^{2} \leq\left[\left\|w^{\prime}(0)\right\|_{2}^{2}+l\|\nabla w(0)\|_{2}^{2}+\left\|\eta_{1}(0)-\eta_{2}(0)\right\|_{g, V}^{2}\right] \mathrm{e}^{m t}
$$

So we get $w(t) \equiv 0$, the uniqueness is proved.

Theorem 2. Let $X$ be a Banach space, and $\{S(t)\}(t \geq 0)$ are the semigroup operator on $X$. $S(t): X \rightarrow X$, $S(t) S(\tau)=S(t+\tau), S(0)=I$, here $I$ is a unit operator. Set $S(t)$ satisfy the follow conditions.

1) $S(t)$ is bounded, namely $\forall R>0,\|u\|_{X} \leq R$, it exists a constant $C(R)$, so that

$$
\|S(t) u\|_{X} \leq C(R)(t \in[0,+\infty))
$$

2) It exists a bounded absorbing set $B_{0} \subset X$, namely, $\forall B \subset B_{0}$, it exists a constant $t_{0}$, so that

$$
S(t) B \subset B_{0}\left(t \geq t_{0}\right)
$$

3) When $t>0, S(t)$ is a completely continuous operator $A$.

Therefore, the semigroup operators $S(t)$ exist a compact global attractor.

Theorem 3. Under the assume of Theorem 1, equations have global attractor

$$
A=\omega\left(B_{0}\right)=\bigcap_{s \geq 0} S(t \geq s) B_{0}
$$

where $B_{0}=\left\{(u, v) \in H_{0}^{2} \times H^{1}:\|(u, v)\|_{H_{0}^{2} \times H^{1}}^{2}=\|u\|_{H_{0}^{2}}^{2}+\|v\|_{H^{1}}^{2} \leq E_{0}+E_{1}\right\}, \quad B_{0} \quad$ is the bounded absorbing set of $H^{2}(\Omega) \times H_{0}^{1}(\Omega)$ and satisfies

1) $S(t) A=A, t>0$;

2) $\lim _{t \rightarrow \infty} \operatorname{dist}\left(S(t) B_{0}, A\right)=0$, here $B \subset H_{0}^{2}(\Omega) \times H^{1}(\Omega)$ and it is a bounded set,

$$
\operatorname{dist}(X, Y)=\operatorname{supinf}_{x \in X}\|x-y\|_{H^{2} \times H^{1}}
$$

Proof. Under the conditions of Theorem 1, it exists the solution semigroup $S(t)$, here $X=H_{0}^{2}(\Omega) \times H^{1}(\Omega)$, $S(t): H^{2} \times H^{1} \rightarrow H^{2} \times H^{1}$.

1) From Lemma 1 to Lemma 2, we can get that $\forall B \subset H_{0}^{2}(\Omega) \times H^{1}(\Omega)$ is a bounded set that includes in the ball $\left\{\|(u, v)\|_{H_{0}^{2} \times H^{1}} \leq R\right\}$,

$$
\left\|S(t)\left(u_{0}, v_{0}\right)\right\|_{H^{2} \times H_{0}^{1}}^{2}=\|u\|_{H^{2}}^{2}+\|v\|_{H_{0}^{1}}^{2} \leq\left\|u_{0}\right\|_{H^{2}}^{2}+\left\|v_{0}\right\|_{H_{0}^{1}}^{2}+C \leq R^{2}+C, \quad\left(t \geq 0,\left(u_{0}, v_{0}\right) \in B\right)
$$

This shows that $S(t)(t \geq 0)$ is uniformly bounded in $H^{2}(\Omega) \times H_{0}^{1}(\Omega)$.

2) Furthermore, for any $\left(u_{0}, v_{0}\right) \in H^{2}(\Omega) \times H^{1}(\Omega)$, when $t \geq \max \left\{t_{1}, t_{2}\right\}$, we have

$$
\left\|S(t)\left(u_{0}, v_{0}\right)\right\|_{H_{0}^{2} \times H^{1}}^{2}=\|u\|_{H_{0}^{2}}^{2}+\|v\|_{H^{1}}^{2} \leq E_{0}+E_{1}
$$

So, we get $B_{0}$ is the bounded absorbing set.

3) Since $H^{2}(\Omega) \times H_{0}^{1}(\Omega) \rightarrow H_{0}^{1}(\Omega) \times L^{2}(\Omega)$ is tightly embedded, which means that the bounded set in $H^{2}(\Omega) \times H_{0}^{1}(\Omega)$ is the tight set in $H_{0}^{1}(\Omega) \times L^{2}(\Omega)$, so the semigroup operator $S(t)$ is completely continuous.

So, the semigroup operators $S(t)$ exist a compact global attractor $A$. The proof is completed.

\section{Acknowledgements}

The authors express their sincere thanks to the anonymous reviewer for his/her careful reading of the paper, giving valuable comments and suggestions. These contributions greatly improved the paper. 


\section{Funding}

This work is supported by the National Natural Sciences Foundation of People’s Republic of China under Grant 11161057.

\section{References}

[1] Messaoudi, S.A. (2003) Blow Up and Global Existence in a Nonlinear Viscoelastic Wave Equation. Mathematische Nachrichten, 260, 58-66. http://dx.doi.org/10.1002/mana.200310104

[2] Cavalcanti, M.M., Domingos Cavalcanti, V.N. and Ferreira, J. (2001) Existence and Uniform Decay for Nonlinear Viscoelastic Equation with Strong Damping. Mathematical Methods in the Applied Sciences, 24, 1043-1053. http://dx.doi.org/10.1002/mma.250

[3] Berrimi, S. and Messaoudi, S.A. (2006) Existence and Decay of Solutions of a Viscoelastic Equation with a Nonlinear Source. Nonlinear Analysis: Theory, Methods \& Applications, 64, 2314-2331. http://dx.doi.org/10.1016/j.na.2005.08.015

[4] Lu, L.Q. and Li, S.J. (2011) Cauchy Problem for a Nonlinear Viscoelastic Equation with Nonlinear Damping and Source Term. Applied Mathematics Letters, 24, 1275-1281. http://dx.doi.org/10.1016/j.aml.2011.01.009

[5] Kafini, M. and Mustafa, M.I. (2014) Blow-Up Result in a Cauchy Viscoelastic Problem with Strong Damping and Dispersive. Nonlinear Analysis: Real World Applications, 20, 14-20. http://dx.doi.org/10.1016/j.nonrwa.2014.04.005

[6] Araújo, R.O., Ma, T. and Qin, Y.M. (2013) Long-Time Behavior of a Quasilinear Viscoelastic Equation with Past History. Journal of Differential Equations, 254, 4066-4087. http://dx.doi.org/10.1016/j.jde.2013.02.010

[7] Qin, Y.M., Feng, B.W. and Zhang, M. (2014) Uniform Attractors for a Non-Autonomous Viscoelastic Equation with a Past History. Nonlinear Analysis: Theory, Methods \& Applications, 101, 1-15. http://dx.doi.org/10.1016/j.na.2014.01.006

[8] Qin, Y.M., Zhang, J.P. and Sun, L.L. (2013) Upper Semicontinuity of Pull Back Attractors for a Non-Autonomous Viscoelastic Equation. Applied Mathematics and Computation, 223, 362-376. http://dx.doi.org/10.1016/j.amc.2013.08.034

[9] Conti, M. and Geredell, P.G. (2015) Existence of Smooth Global Attractors for Nonlinear Viscoelastic Equations with Memory. Journal of Evolution Equations. http://dx.doi.org/10.1007/s00028-014-0270-2

[10] Cavalcanti, M.M., Domingos Cavalcanti, V.N., Prates Filho, J.A. and Soriano, J.A. (2001) Existence and Uniform Decay Rates for Viscoelastic Problems with Non-Linear Boundary Damping. Differential and Integral Equations, 14, 85116.

[11] Muñoz Rivera, J.E., Lapa, E.C. and Barreto, R. (1996) Decay Rates for Viscoelastic Plates with Memory. Journal of Elasticity, 44, 61-87. http://dx.doi.org/10.1007/BF00042192

[12] Wu, S.T. (2011) General Decay of Solutions for a Viscoelastic Equation with Nonlinear Damping and Source Terms. Acta Mathematica Scientia, 31, 1436-1448. http://dx.doi.org/10.1016/S0252-9602(11)60329-9

[13] Ma, T.F. and Narciso, V. (2010) Global Attractor for a Model of Extensible Beam with Nonlinear Damping and Source Terms. Nonlinear Analysis: Theory, Methods \& Applications, 73, 3402-3412. http://dx.doi.org/10.1016/j.na.2010.07.023

[14] Qin, Y., Deng, S. and Schulze, W. (2009) Uniform Compact Attractors for a Nonlinear Non-Autonomous Equation of Viscoelasticity. Journal of Partial Differential Equations, 22, 153-198. 